\title{
BMJ Open Implementation of 'Goals of Patient Care' medical treatment orders in residential aged care facilities: protocol for a randomised controlled trial
}

\author{
Ruth S Martin, ${ }^{1,2}$ Barbara J Hayes, ${ }^{1}$ Anastasia Hutchinson, ${ }^{1,3}$ Paul Yates, ${ }^{2,4}$ \\ Wen Kwang Lim ${ }^{1,2,5}$
}

To cite: Martin RS, Hayes BJ, Hutchinson A, et al. Implementation of 'Goals of Patient Care' medical treatment orders in residential aged care facilities: protocol for a randomised controlled trial. BMJ Open 2017;7:e013909.

doi:10.1136/bmjopen-2016013909

- Prepublication history and additional material is available. To view please visit the journal (http://dx.doi.org/ 10.1136/bmjopen-2016013909).

Received 17 August 2016 Revised 12 December 2016 Accepted 2 February 2017

CrossMark

${ }^{1}$ Northern Health, Epping, Victoria, Australia

${ }^{2}$ University of Melbourne, Melbourne, Victoria, Australia ${ }^{3}$ Deakin University, Geelong, Victoria, Australia

${ }^{4}$ Austin Health, Heidelberg,

Victoria, Australia

${ }^{5}$ Melbourne Health,

Melbourne, Victoria, Australia

Correspondence to

Dr Ruth Martin;

Ruth.martin@nh.org.au

\section{ABSTRACT}

Introduction: Systematic reviews demonstrate that advance care planning (ACP) has many positive effects for residents of aged care facilities, including decreased hospitalisation. The proposed Residential Aged Care Facility (RACF) 'Goals of Patient Care' (GOPC) form incorporates a resident's prior advance care plan into medical treatment orders. Where none exists, it captures residents' preferences. This documentation helps guide healthcare decisions made at times of acute clinical deterioration.

Methods and analysis: This is a mixed methods study. An unblinded cluster randomised controlled trial is proposed in three pairs of RACFs. In the intervention arm, GOPC forms will be completed by a doctor incorporating advance care plans or wishes. In the control arm, residents will have usual care which may include an advance care plan. The primary hypothesis is that the GOPC form is superior to standard ACP alone and will lead to decreased hospitalisation due to clearer documentation of residents' medical treatment plans. The primary outcome will be an analysis of the effect of the GOPC medical treatment orders on emergency department attendances and hospital admissions at 6 months. Secondary outcome measurements will include change in hospitalisation rates at 3 and 12 months, length of stay and external mortality rates among others. Qualitative interviews, 12 months post GOPC implementation, will be used for process evaluation of the GOPC and to evaluate staff perceptions of the form's usefulness for improving communication and medical decision-making at a time of deterioration. Dissemination: The results will be disseminated in peer review journals and research conferences. This robust randomised controlled trial will provide highquality data about the influence of medical treatment orders that incorporate ACP or preferences adding to the current gap in knowledge and evidence in this area. Trial registration number: ACTRN12615000298516, Results.

\section{INTRODUCTION}

Goals of Patient Care (GOPC) form

The trial Residential Aged Care Facility (RACF) GOPC form (see online

\section{Strengths limitations of this study}

New medical treatment order, specifically for aged care facility residents.

- First randomised controlled trial examining the effects of the Goals of Patient Care (GOPC) process in Residential Aged Care Facilities.

- Quantitative and qualitative methods for thorough examination of the GOPC medical treatment orders' effects.

- Small number of residential aged care facilities involved.

- The GOPC is not evaluated in this study for its effect on improving compliance of medical treatment with the residents' treatment preferences.

supplementary appendix 1) is a document used to record medical treatment plans for residents in the event of clinical deterioration. It takes into account the current medical condition as well as residents' wishes and any prior advance care planning (ACP). As it is specifically for residents in RACFs, it identifies whether residents are open to hospital transfer for treatment escalation. The form is completed by a physician with the resident or their substitute medical decisionmaker (SDM), or both.

The GOPC form originated in Tasmania, Australia, where it was developed for their inpatient and RACF populations. ${ }^{1}$ This approach identifies: (1) the overall goals of care; and (2) specific treatment escalation and limitations proportionate to that goal. The aim is to avoid focusing only on interventions in isolation, such as cardiopulmonary resuscitation (CPR), intubation or intravenous antibiotics. In 2013, this approach was adapted by one of the authors (BH) to replace the hospital 'limitation of medical treatment' form in use by Northern Health, Victoria. ${ }^{2}$ From this, BH developed the trial version of the GOPC specifically for RACF residents addressing limitations to 
treatment and place of care. The form was developed in consultation with geriatricians working with the RACF inreach service. This is the first study examining its effects on RACF residents. The form has been made available to other health services in the state of Victoria and there are plans for its wider use.

There are three overall goals with six potential goal options, see figure 1:

- Goal A and B apply to residents for whom the plan is to treat reversible illness, even if the burdens of that treatment might be considerable; hospital transfer would be appropriate. Goal A identifies residents for no treatment limitation and for whom attempted CPR would apply. Goal B identifies residents for whom some treatment limitations apply, including not for attempted CPR or intubation.

- Goal C applies to residents for whom investigations or treatment should only be undertaken if nonburdensome. Goal C1 identifies residents for trial of treatment at facility and for hospital transfer if required. Goal C2 identifies residents for trial of treatment at the facility but not for hospital transfer in the event of deterioration. Goal C3 identifies residents who are not for further treatments of new illnesses, and who are opting for symptom management only.
- Goal D identifies residents who are in the terminal stage of illness (last hours and days of life), and for whom all interventions should be for comfort only.

The GOPC form is different from, but related to, an advance care plan. An advance care plan is usually regarded as a communique between residents or their SDM and staff, and is completed by the resident/SDM. The GOPC, however, is a communique between staff and is completed by a doctor. Using a shared decisionmaking discussion with the resident and/or SDM, information about the resident's illness trajectory, potential for deterioration and medical management options is provided. Within this context, prior ACP is translated into clinical language to guide healthcare professionals in their treatment decisions for that resident. In the absence of formal prior ACP, medical treatment planning can still take place by exploring, and taking into account, the resident's values and what matters most to them. This can be done with a resident who retains capacity or with the SDM of a resident lacking capacity to participate. Availability of a GOPC form can be particularly helpful when a resident is being reviewed by a doctor or nurse who is unfamiliar with that person, their values or their treatment plans. Availability of a

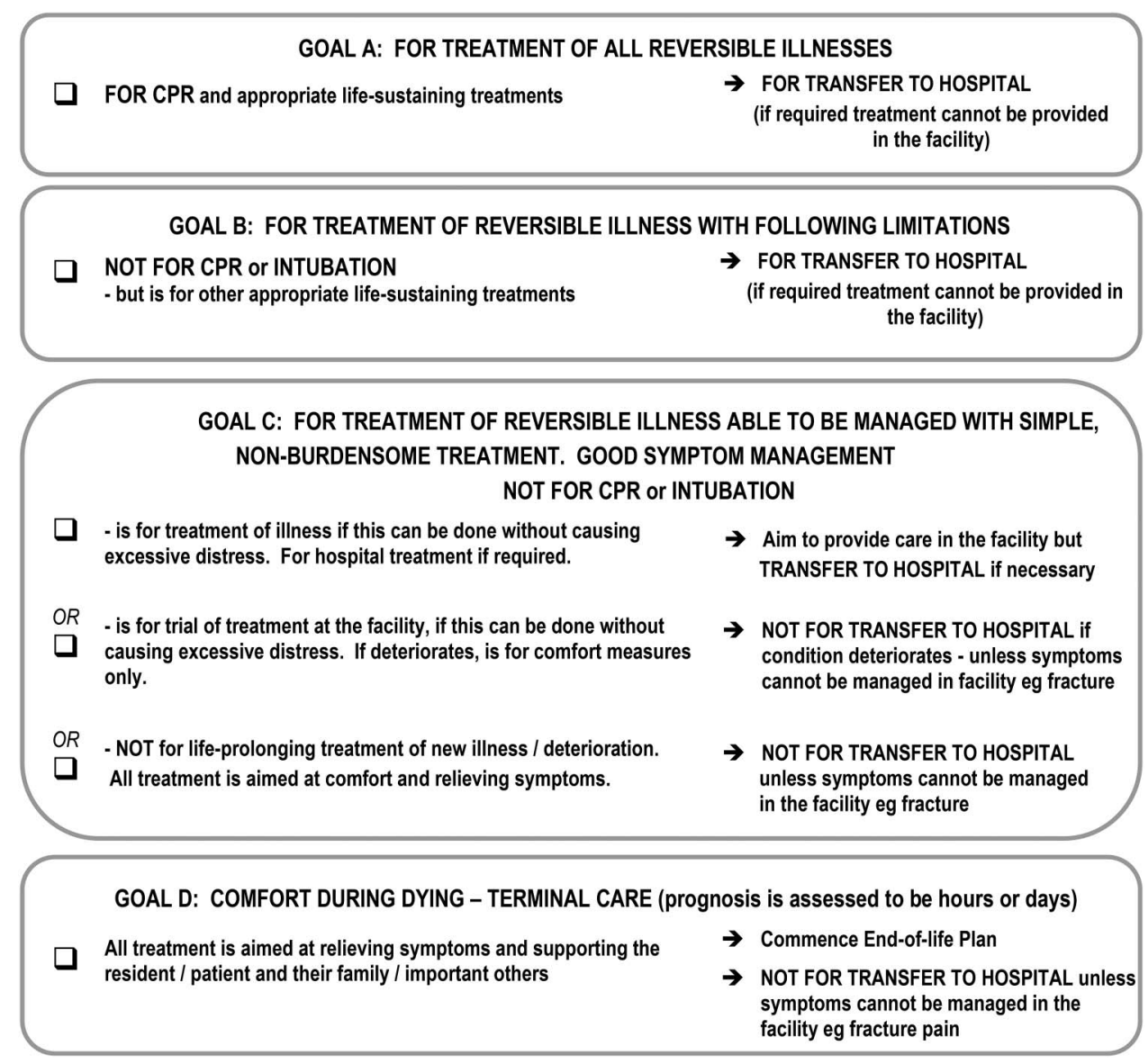

Figure 1 The options on the Goals of Patient Care medical treatment orders as seen on the complete Goals of Patient Care Residential Aged Care Facility form are shown here. 
completed GOPC form is not intended to replace a discussion with the SDM at the time of deterioration. It does provide a starting point for that discussion by a clinician who does not know the resident and can be particularly helpful when the SDM is unable to be contacted in a timely way. Additionally, the language is unambiguous and directive in nature.

\section{Background}

Systematic review identifies ACP as a beneficial intervention for aged care facility residents. ${ }^{3}$ Studies in the USA have shown improvements in treatment decisions for residents with the introduction of medical treatment forms such as the Physician Orders for Life-Sustaining Treatment (POLST) and others adapted from it. ${ }^{4}$ Such studies have not been conducted in Australia and the intention of this study is to show that such innovations are translatable to our target population. We hypothesise that the introduction of this medical treatment order will lead to decreased acute healthcare usage, when compared with usual care, by improving communication of the residents' wishes to all healthcare staff leading to more appropriate healthcare decisions.

The POLST was first introduced to address shortcomings found with advance care plans, including difficulty with their interpretation ${ }^{5-9}$ and not being in a form that ambulance paramedics could follow. ${ }^{10}$ A systematic review of the literature has shown that extensive ACP interventions have resulted in increased compliance with patient wishes and satisfaction with care, but needs to include more than just a written document. ${ }^{11}$ The POLST intervention, like the GOPC form, was developed to help ensure the wishes of individuals with advanced illness or frailty were honoured by documenting their preferences as medical treatment orders. ${ }^{12}$ Studies have shown that patients with such orders were less likely to receive unwanted interventions including hospitalisation, ${ }^{13-15}$ and intravenous fluids, ${ }^{16}$ than those with traditional ACPs alone. ${ }^{13}$

The incidence of transfers from RACFs to the emergency department (ED) has been measured at $<30$ transfers per 100 bed days, ${ }^{17}$ but varies depending on facility and location. Hospitalisation can be burdensome for nursing home residents, ${ }^{5} 18$ and many, when asked, would prefer to be treated in their RACF where possible. ${ }^{19}$ Given their frailty, high incidence of dementia and multimorbidity RACF residents have an increased incidence of acute illness compared with the ambulatory population. This is reflected by a high incidence of acute healthcare usage. ${ }^{6}$ Up to $48 \%$ of these hospital transfers are thought to be avoidable. ${ }^{72}$ Interventions targeting these admissions, according to a recent systematic review, ${ }^{20}$ include, improving palliative care provision, ${ }^{21-23}$ improving ACP interventions, ${ }^{24} 25$ improving treatment of pneumonia and chronic obstructive pulmonary disease within facilities ${ }^{26-28}$ and providing ambulatory geriatric care through geriatrician review of residents within RACFs. ${ }^{19} 2930$
Dementia, estimated to affect over $50 \%$ of RACF residents, ${ }^{18} 3132$ hinders the decision-making capacity of the resident, especially at times of acute illness. The prevalence of dementia also means that at the time of admission to the RACF, many residents will no longer be able to undertake their own ACP. Local RACF practice for this situation is to invite the SDM to complete an ACP on behalf of the resident, a document that cannot have the same authority as a resident-completed advance care plan. The introduction of the RACF 'GOPC' medical treatment orders will make the wishes of frail residents clearer, but within the parameters of treatment that might be effective for their condition. We hypothesise that the GOPC implementation will result in medical decisions being more congruent with residents' wishes, and more appropriate for residents' medical conditions.

\section{Study objectives}

The primary objective is to show that the introduction of the 'GOPC' medical treatment orders will lead to decreased ED attendances and admissions for RACF residents at 6 months post implementation as compared with usual care, by improving communication of the residents wishes leading to more appropriate usage of acute hospital care.

The secondary objectives are to demonstrate that between intervention and control facilities the intervention will result in:

- a change in the rate of ED attendances, inpatient admissions and acute length of stay at 3 and 12 months;

- a change in acute healthcare usage;

- a change in healthcare costs;

- a change in external mortality rate;

- a change in facilitation of healthcare decision-making for all staff;

- a change in conflict between RACF staff, visiting healthcare professionals, residents and families when there is a need for acute healthcare decisions.

\section{METHODS}

Baseline characteristics and assessments will be documented for all participants. These will include age, sex, English-speaking status, comorbidities, presence of a lifelimiting illness (excluding dementia) and medications. A cognitive screen will be undertaken using the Mini Mental State Examination (MMSE) ${ }^{33}$ and also correlated with a diagnosis of dementia and use of medical treatments for dementia. A functional assessment screen will use the Barthel Index, ${ }^{34}$ depression will be screened for using the Geriatric Depression Scale ${ }^{35}$ frailty will be assessed with Clinical Frailty Scale ${ }^{36}$ and a geriatrician will do a brief capacity assessment. The presence of a prior instructional advance care plan and/or appointment of a SDM (medical enduring power of attorney) will be recorded, if available in the facility notes. 
Hospital usage for each facility will be evaluated by accessing local hospital records to calculate a baseline event rate for this, 3, 6 and 12 months prior to initiation of the study.

The following data will be collected at 3, 6 and 12 months for included participants: acute healthcare usage including ED attendances, emergency admissions, outpatient department (OPD) attendances, residential inreach reviews (ambulatory geriatricians), length of hospital stay (LOS) and the associated costs. Death rates and place of death will also be recorded. 12 months after the implementation of the GOPC, the qualitative evaluation will take place with staff from the intervention facilities. The qualitative aspect of the study will complement the quantitative study and provide evidence that implementation of the GOPC intervention in RACFs is feasible and acceptable to clinicians caring for RACF residents.

Data triangulation between the quantitative and qualitative data will be undertaken to ascertain that the intervention is beneficial from a clinical and a healthcare administration perspective. ${ }^{37} 38$

Focus group interviews will be used for exploring experiences of ACP and the GOPC implementation with RACF staff (excluding doctors). The views of general practitioners (GPs) who visit the intervention facilities will be explored using one-to-one semistructured interviews.

Focus groups and individual interviews will be audio recorded and a question guide will be used to explore with participants: their understanding of ACP; experiences of undertaking and implementing ACP within the RACF; understanding of the purpose and use of the GOPC; experiences of using the GOPC form at a time of resident deterioration and views about the relative usefulness of ACP and GOPC. Qualitative research is iterative and unanticipated themes from earlier interviews will be explored in the later interviews. ${ }^{38} 39$ The focus groups will be facilitated by the principal researcher and an associate researcher trained in qualitative methodology. Individual interviews will be undertaken by the principal researcher.

The recorded interviews will be transcribed and key themes emerging from the interviews will be identified by the principal researcher and a coresearcher on an ongoing basis. Qualitative research is iterative and unanticipated themes from earlier interviews will be explored in the later interviews. ${ }^{38} 39$ The RACF staff focus groups will be repeated until saturation of themes has been reached, it is anticipated that saturation will be achieved with three focus groups, however, if required additional focus groups will be conducted.

A table indicating a schedule table of enrolment, interventions and assessments as is used in Standard Protocol Items; Recommendations for Interventional Trials (SPIRIT) is attached (see online supplementary appendix 2). ${ }^{40}$
Table 1 Baseline characteristics and assessments

\begin{tabular}{|c|c|}
\hline Baseline characteristics & Baseline assessments \\
\hline Sex & $\begin{array}{l}\text { Mini Mental State } \\
\text { Examination }\end{array}$ \\
\hline Age & Barthel Index of function \\
\hline Comorbidities & Clinical Frailty Scale \\
\hline Presence of life-limiting illness & $\begin{array}{l}\text { Geriatric Depression } \\
\text { Scale }\end{array}$ \\
\hline Diagnosis of dementia & Capacity \\
\hline Dementia treatment & \\
\hline Regular medications & \\
\hline PRN medications & \\
\hline English as first language & \\
\hline Advance care plan & \\
\hline Medical power of attorney & \\
\hline $\begin{array}{l}\text { Evidence medical power of } \\
\text { attorney }\end{array}$ & \\
\hline
\end{tabular}

\section{Baseline characteristics and assessments}

Baseline characteristics of participants were gathered and baseline assessments performed as outlined in table 1 .

\section{Study design}

The study design, see figure 2 , is an unblinded prospective cluster randomised controlled trial evaluating the effects of the implementation of the GOPC medical treatment orders for RACF residents. The clusters are defined as the individual RACFs. The RACFs are organised into cluster pairs and then randomised at a facility level.

\section{Participants}

The study population is all residents within the six participating RACFs for whom written informed consent can be obtained. A total of 45 facilities in the area were invited to partake by email contact followed up with a phone call to the facility manager. For those agreeable, a meeting took place to explain the study and confirm willingness to participate. Written informed consent form the facility manager was then obtained so as to access the RACFs prior 12-month hospital usage rates from local health services as well as basic demographic information. Of the 45 facilities, eight agreed to participate. Two withdrew consent due higher management of the aged care group not wanting to partake. The six remaining facilities were matched on key characteristics and randomised. Individual recruitment of residents then took place in each participating facility.

Healthcare staff will be invited to take part in focus groups and individual interviews by personal invitation. Staff across a range of positions within the facilities will be included.

\section{Inclusion criteria}

All residents in the age care facilities participating in the study, together with their SDM, will be invited to participate. 


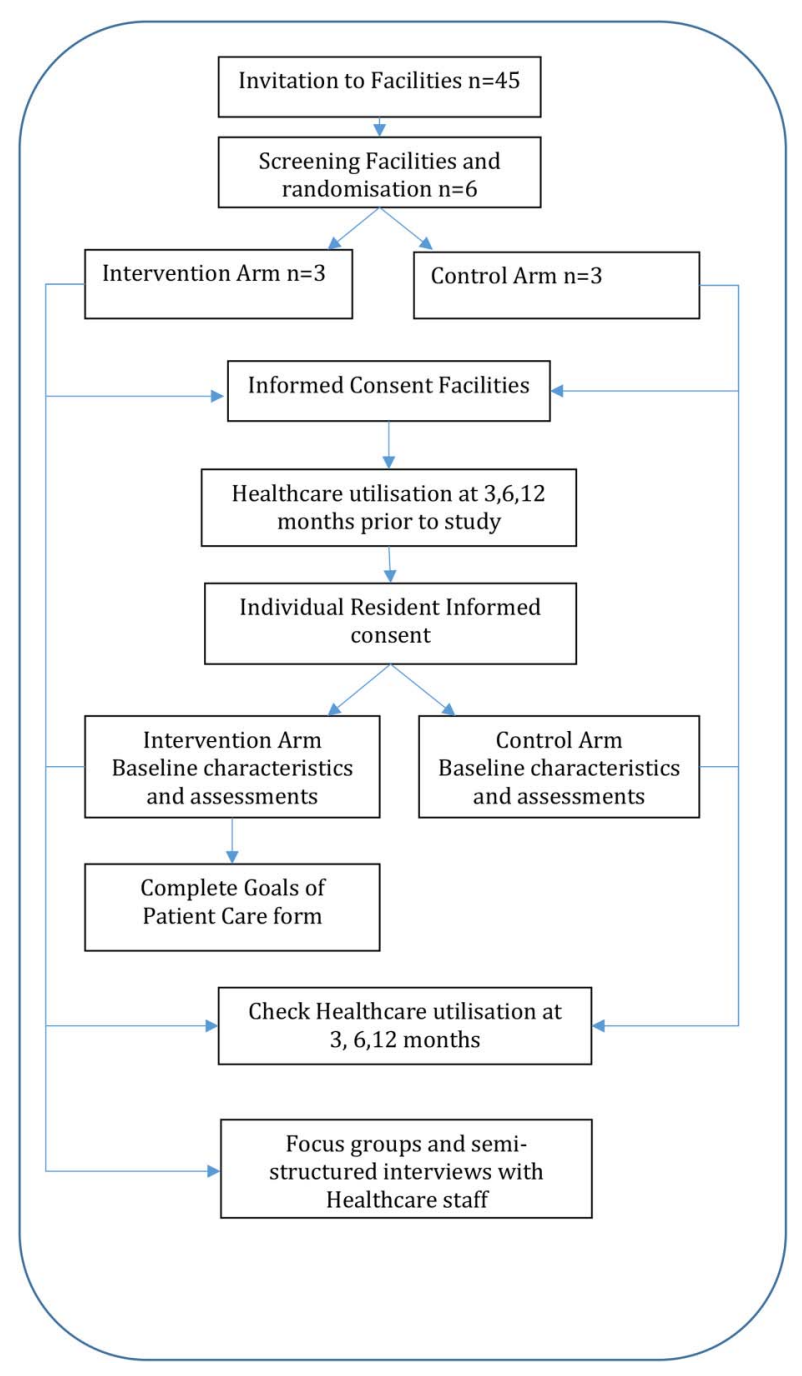

Figure 2 The study design is outlined from point of recruitment through to implementation and quantitative and qualitative data collection.

\section{Exclusion criteria}

Residents who lack capacity to provide written informed consent will be excluded from participating in our study, unless they have a SDM who is able to participate in the study in conjunction with or on behalf of a resident lacking medical decision-making capacity.

\section{Consent}

Participation in the study by individual residents, SDMs and staff is voluntary. Written informed consent will be obtained from the management of the RACFs involved. Written informed consent will be obtained from all participants in the intervention and control group. In event of decreased or a definite lack of capacity, cosigning/ substitute signing of the consent form by the SDM will be obtained. Telephone consent will be obtained from those SDMs that cannot attend in person (anticipating frailty issues with partners of residents) but wish to be involved. Telephone consent will be witnessed by a second person. A participant information sheet and consent form and a sample copy of the GOPC form will be mailed to those persons from whom telephone consent will be sought.

For the healthcare professionals who participate in the study, written informed consent will be obtained prior to participation in focus group or individual interviews.

\section{Intervention}

The interventions to be compared are that of the new GOPC medical treatment order form and discussion, and usual care. It is important to note that immediately prior to this study there has been an extensive ACP and palliative care education initiative for local RACF staff using standardised content. This was an Australian Government and Advance Care Planning Australia initiative known as 'Decision Assist'. ${ }^{41}$

The GOPC form, as described in the introduction, is a medical treatment order completed by a doctor in collaboration with the resident or their SDM. This will occur in addition to any ACP already being undertaken by the RACF staff in the intervention sites. The GOPC indicates the preferred course of action in the event of clinical deterioration. It will be placed in the residents notes in their section on ACP. It will be available to all healthcare professionals reviewing the resident and a copy will be transferred with them to the ED with their RACF documentation. In case of computerised medical notes, the document will be scanned on to the system to the ACP section.

\section{Usual care}

'Usual care' will include the current processes in use within the individual RACFs. For many residents, this will include an advance care plan, which should be present in their paper or computerised notes. These advance care plans are sometimes completed by the resident and/or their SDM alone, without input from health professionals. In some facilities the RACF staff is involved in the ACP discussion and form completion. In others, the GPs are either required to be involved in the discussion or simply to sign the completed form. In no facilities will medical treatment orders be in use, as they are not currently used anywhere in local health services. Not all residents will be expected to have an advance care plan but it is expected that all will have been invited to complete an advance care plan at some stage since admission to the RACF.

\section{Outcome measures}

The primary outcome measure is that providing residents with a 'GOPC' medical treatment order will result in a $40 \%$ decrease in emergency attendance and emergency hospital admission at 6 months compared between intervention and control facilities.

- Secondary outcome measures will include:

- acute healthcare usage at 3,6 and 12 months (ED attendances, acute care admissions, acute care length of stay, total inpatient bed days and number of ambulatory care attendances); 
- direct costs of acute healthcare usage;

- the rate of uptake of the GOPC by residents in intervention RACFs

- the number of changes made to GOPC over 12 months;

- the presence of a diagnosis of Dementia with associated MMSE score and medical treatments on recruitment;

- 12-month mortality rate and place of death.

Qualitative outcomes

- The staff/resident/SDM opinion on improved communication of residents' healthcare wishes;

- the staff opinion on effect of GOPC on healthcare decision-making;

- staff/resident/SDM opinion on decreased conflict between RACF staff, visiting healthcare professionals, residents and families at times of acute healthcare decision-making.

\section{Sample size}

On calculation for individual randomisation for this study, $\mathrm{n}=157$ persons per period for each arm were given a significance of 0.05 and $80 \%$ power. On calculation for cluster randomisation given an anticipated event rate of 0.5 (emergency reviews or admissions $/ 6$ months/facility bed) in control and 0.3 in intervention facilities and assumed intracluster correlation $(p)$ which is a combination of within cluster variance, of 0.01 the estimated number of clusters required per intervention and control strata is 3.5. On testing feasibility of three clusters, it was found to be feasible if the number of clusters (k) was $>$ n (157) $\times \mathrm{p}(0.011)$. The anticipated event rates were based on a prior randomised controlled trial where the level of reduction in hospitalisation was in this range. $^{24}$

\section{Randomisation}

Randomisation will use the add-in random allocation program 'ralloc' available in Stata V.12.1 (StataCorp LP, Texas, USA). The randomisation will occur at facility level to minimise contamination between residents within the same facility. Facilities will be organised into cluster pairs based on their prior 12-month event rate for hospital attendances and admissions. Facilities will be blinded to the random allocation prior to agreeing to participate. On randomisation, no further blinding will be undertaken.

\section{STATISTICAL METHODS}

\section{Quantitative data analysis}

Descriptive statistics will be used to compare healthcare usage rates, and other secondary outcomes, between the intervention and the control arms at 3, 6 and 12 months. Multilevel Poisson regression models will be established to account for the intraclass correlation within each RACF when assessing the primary outcome of healthcare usage rates. Appropriate parametric and non-parametric continuous data statistical tests and $\chi^{2}$ tests will be used to evaluate the effectiveness of the intervention for the secondary outcomes. Descriptive statistics will also be reported at baseline to demonstrate the consistency of healthcare usage between the intervention and control arms prior to the study intervention. A table of statistical methods used for each outcome has been made (see online supplementary appendix 3 ).

\section{Qualitative data analysis}

The transcribed focus group and individual interviews will be transcribed verbatim. Transcribed data will be analysed thematically, using open and axial coding. ${ }^{38}$ The coding will be undertaken by two researchers independently. Findings from the qualitative data will be analysed using qualitative description. ${ }^{42}$ Triangulation of findings from the qualitative analysis will be applied to the quantitative analysis to better understand, and interpret, the quantitative findings.

\section{DISCUSSION}

This study protocol is the first randomised controlled trial examining the effect of a GOPC medical treatment order in RACFs. Clinical studies have previously shown positive effects of ACP, particularly when translated into medical treatment orders, ${ }^{12} 1343$ in the RACF population. Owing to lack of high quality studies in the area, the evidence is mainly taken from pooled low-quality publications. $^{3}$

This study will perform a cluster randomised controlled trial in the area to provide the required data on medical treatment order effects in the RACF population. This trial design will allow for clustering of sites with similar key baseline characteristics thus limiting the intracluster variance and allowing for better comparison. By clustering residents by site, contamination of effect between residents in the same facility will be minimised. By using a control arm, it will be possible to examine and compare the effect of the intervention versus that of usual care. By minimising exclusion criteria, it is expected that a representative sample of all nursing home residents will be recruited for the study.

Hospitalisation has been chosen as the primary outcome measure for this study as it is well described as a positive effect of other types of ACP. ${ }^{12} 24254344$ Open communication regarding residents' wishes can lead to a decrease in unwanted acute hospitalisation. ${ }^{6}$ Given the frailty of this population, a 6-month period for the primary outcome was judged as most appropriate, with additional assessments at 3 and 12 months to provide a clearer picture of event rates over time. The GOPC form clearly states whether residents are open to a trial of treatment in the facility and if they wish for hospital transfer for treatment escalation if not improving. The clear language should avoid ambiguity and should help staff more easily decide on a treatment plan according to the prior choices made on the form. 
Death rates and place of death are being examined to identify whether the form leads to a greater number of residents dying within the facility, which is the preference of the majority of residents and their SDMs. ${ }^{45}$ Prior studies have shown that ACP can increase the rates of residents dying in their home by $29-40 \% .^{25} 4345{ }^{46}$ This study will examine whether similar rates are achieved through introduction of the GOPC form.

Evaluation of the situations in which the forms were used by staff will occur through the focus groups and semistructured interviews. Additionally, the effect the GOPC form had on the decisions made for residents when they became unwell will be explored, together with whether the decisions made were consistent with the medical treatment plan documented on the form. It is expected that the GOPC form, with clearly stated intentions for treatment, will help decision-making at a time of clinical deterioration and decrease conflict between healthcare staff. There is rich information about use of the form that can only be identified through this qualitative analysis. It is expected that the reported experiences of nursing staff, management staff and GPs with ACP, and with the GOPC form, will provide valuable insights about the use of medical treatment orders in RACFs.

Limitations in the study include a small number of included RACFs; it would provide further confidence in the results to repeat it with an increased sample size. The primary outcome is hospitalisation rather than congruency with wishes, which is a secondary outcome; however, due to an inability to accurately identify all the times in which actions would be congruent with wishes as well as not, it was felt hospitalisation would be a more accurate observation. The reasons for any identified hospitalisations against proposed wishes will then be reviewed.

\section{CONCLUSION}

The GOPC medical treatment orders are an innovation in the field of ACP. It is anticipated that this robust examination, using quantitative and qualitative methodologies, will demonstrate their implementation to have beneficial effects for residents, RACFs and health services.

Acknowledgements The authors acknowledge Northern Health Foundation for a small research grant for the study, and Northern Health Aged Care Research Department and The University of Melbourne for research scholarships for the principal researcher.

Contributors All authors, RSM, BJH, AH, PY and WKL were involved in the conception of the study. RSM and WKL were responsible for recruitment. BJH developed the GOPC form. AH was involved in the statistical planning. PY was involved in the ethics applications. RSM, BJH, AH, PY and WKL were involved in drafting the work. RSM, BJH, AH, PY and WKL have approved the final version for print. RSM, BJH, AH, PY and WKL agree to be accountable for all aspects of the work.

Funding Northern Health Foundation.

Competing interests None declared.
Ethics approval The trial has ethical approval from the Northern Health Human Research Ethics Committee (HREC/15/NH/6). For retrieval of baseline hospital usage rates two further ethics approvals were sought. Approval was given from the Austin Health Human Research Ethics Committee; LNR 15/ Austin/169. Approval was also given from the Melbourne Health Quality Assurance section of the Ethics Committee; QA2016047.

Provenance and peer review Not commissioned; externally peer reviewed.

Data sharing statement Sharing of data from the project will be carried out through research publications and presentations at local, national and international meetings.

Open Access This is an Open Access article distributed in accordance with the Creative Commons Attribution Non Commercial (CC BY-NC 4.0) license, which permits others to distribute, remix, adapt, build upon this work noncommercially, and license their derivative works on different terms, provided the original work is properly cited and the use is non-commercial. See: http:// creativecommons.org/licenses/by-nc/4.0/

\section{REFERENCES}

1. Thomas RL, Zubair MY, Hayes B, et al. Goals of care: a clinical framework for limitation of medical treatment. Med $J$ Aust 2014;201:452-5.

2. Brimblecombe C, Crosbie D, Lim WK, et al. The Goals of Patient Care project: implementing a proactive approach to patient-centred decision-making. Intern Med J 2014;44:961-6.

3. Martin RS, Hayes B, Gregorevic K, et al. The effects of advance care planning interventions on nursing home residents: a systematic review. J Am Med Dir Assoc 2016;17:284-93.

4. Center for Ethics in Health Care, Oregon Health \& Science University. The physician orders for life-sustaining treatment program (POLST). http://www.ohsu.edu/polst

5. Happ MB, Capezuti E, Strumpf NE, et al. Advance care planning and end-of-life care for hospitalized nursing home residents. $J$ Am Geriatr Soc 2002;50:829-35.

6. Kayser-Jones JS, Wiener CL, Barbaccia JC. Factors contributing to the hospitalization of nursing home residents. Gerontologist 1989;29:502-10.

7. Fagerlin A, Schneider CE. Enough: The failure of the living will. Hastings Center Rep 2004;34:30-42.

8. Hickman SE, Hammes BJ, Moss AH, et al. Hope for the future: achieving the original intent of advance directives. Hastings Center Rep 2005;35:S26-30

9. Teno J, Lynn J, Wenger N, et al. Advance directives for seriously ill hospitalized patients: effectiveness with the patient self-determination act and the SUPPORT intervention. J Am Geriatr Soc 1997;45:500-7.

10. Marco CA, Schears RM. Prehospital resuscitation practices: a survey of prehospital providers. J Emerg Med 2003;24:101-6.

11. Brinkman-Stoppelenburg A, Rietjens JA, Van der Heide A. The effects of advance care planning on end-of-life care: a systematic review. Palliat Med 2014;28:1000-25.

12. Tolle SW, Tilden VP, Nelson CA, et al. A prospective study of the efficacy of the physician order form for life-sustaining treatment. J Am Geriatr Soc 1998;46:1097-102.

13. Hickman SE, Nelson CA, Perrin NA, et al. A comparison of methods to communicate treatment preferences in nursing facilities: traditional practices versus the physician orders for life-sustaining treatment program. J Am Geriatr Soc 2010;58:1241-8.

14. Lee MA, Brummel-Smith K, Meyer J, et al. Physician orders for life-sustaining treatment (POLST): outcomes in a PACE program J Am Geriatr Soc 2000;48:1219-25.

15. Gillick MR. Adapting advance medical planning for the nursing home. J Palliat Med 2004;7:357-61.

16. Dunn PM, Schmidt TA, Carley MM, et al. A method to communicate patient preferences about medically indicated life-sustaining treatment in the out-of-hospital setting. J Am Geriatr Soc 1996;44:785-91.

17. Arendts $\mathrm{G}$, Howard $\mathrm{K}$. The interface between residential aged care and the emergency department: a systematic review. Age Ageing 2010;39:306-12.

18. Ervin K, Finlayson S, Cross M. The management of behavioural problems associated with dementia in rural aged care. Collegian 2012;19:85-95.

19. Harvey $\mathrm{P}$, Storer M, Berlowitz DJ, et al. Feasibility and impact of a post-discharge geriatric evaluation and management service for patients from residential care: the Residential Care Intervention Program in the Elderly (RECIPE). BMC Geriatr 2014;14:48. 
20. Graverholt B, Forsetlund L, Jamtvedt G. Reducing hospital admissions from nursing homes: a systematic review. BMC Health Serv Res 2014;14:36

21. Casarett D, Karlawish J, Morales $\mathrm{K}$, et al. Improving the use of hospice services in nursing homes. JAMA 2005;294:211-7.

22. Hanson LC, Reynolds KS, Henderson M, et al. A quality improvement intervention to increase palliative care in nursing homes. J Palliat Med 2005;8:576-84.

23. Kovach CR, Wilson SA, Noonan PE. The effects of hospice interventions on behaviors, discomfort, and physical complications of end stage dementia nursing home residents. American Journal of Alzheimer's Disease and Other Dementias 1996;11:7-15.

24. Molloy DW, Guyatt GH, Russo R, et al. Systematic implementation of an advance directive program in nursing homes: a randomized controlled trial. JAMA. 2000;283:1437-44.

25. Caplan GA, Meller A, Squires B, et al. Advance care planning and hospital in the nursing home. Age Ageing. 2006;35:581-5.

26. Hutt E, Ruscin JM, Linnebur SA, et al. A multifaceted intervention to implement guidelines Did Not affect hospitalization rates for nursing home-acquired pneumonia. J Am Med Dir Assoc 2011;12:499-507.

27. Loeb M, Carusone SC, Goeree R, et al. Effect of a clinical pathway to reduce hospitalizations in nursing home residents with pneumonia: a randomized controlled trial. JAMA 2006:295:2503-10.

28. Lee DT, Lee IF, Mackenzie AE, et al. Effects of a care protocol on care outcomes in older nursing home patients with chronic obstructive pulmonary disease. J Am Geriatr Soc 2002;50:870-6.

29. Schippinger W, Hartinger G, Hierzer A, et al. Mobiler geriatrischer konsiliardienst für pflegeheime: untersuchung der effektivität eines internistisch-fachärztlichen konsiliardienstes zur medizinischen versorgung von pflegeheimbewohnern (originalien). Z Gerontol Geriatr 2012;45:735-41.

30. Díaz-Gegúndez M, Paluzie G, Sanz-Ballester C, et al. Evaluación de un programa de intervención en residencias geriátricas para reducir la frecuentación hospitalaria. Rev Esp Geriatr Gerontol 2011;46: 261-4.

31. Daly JM, Bay CP, Levy BT, et al. Caring for people with dementia and challenging behaviours in nursing homes: a needs assessment geriatric nursing. Geriatr Nurs 2015;36:182-91.

32. Australian Institute of Health and Welfare (AIHW). Residential aged care in Australia 2009-10: a statistical overview. Aged care statistics series no. 35. Cat. no. AGE 66. Canberra: Australian Institute of Health and Welfare (AIHW), 2011.

33. Folstein MF, Folstein SE, McHugh PR. "Mini-mental state". A practical method for grading the cognitive state of patients for the clinician. J Psychiatr Res 1975;12:189-98.

34. Mahoney FI, Barthel DW. Functional evaluation: the Barthel Index. Md State Med J 1965;14:61-5.

35. Brown LM, Schinka JA. Development and initial validation of a 15 -item informant version of the Geriatric Depression Scale. Int J Geriat Psychiatry 2005;20: 911-8.

36. Rockwood K, Song X, MacKnight C, et al. A global clinical measure of fitness and frailty in elderly people. CMAJ 2005;173:489-95.

37. Morgan D. Practical strategies for combining qualitative and quantitative methods: applications to health research. Qualitative Health Research 1998;8:362-76.

38. Liamputtong P, Ezzy D. Qualitative research methods. Melbourne, VIC: Oxford University Press, 2005:404. ISBN 019551744X.

39. Starks H, Trinidad SB. Choose your method: a comparison of phenomenology, discourse analysis, and grounded theory. Qual Health Res 2007;17:1372-80.

40. http://www.spirit-statement.org/publications-downloads/ (accessed 8 Nov 2016)

41. https://www.caresearch.com.au/Caresearch/tabid/2583/Default.aspx (accessed 14 Nov 2016)

42. Sandelowski M. Focus on research methods-whatever happened to qualitative description?. Research in nursing and health 2000;23:334-40.

43. Levy C, Morris M, Kramer A. Improving end-of-life outcomes in nursing homes by targeting residents at high-risk of mortality for palliative care: program description and evaluation. $J$ Palliat Med 2008;11:217-25.

44. Zweig SC, Kruse RL, Binder EF, et al. Effect of do-not-resuscitate orders on hospitalization of nursing home residents evaluated for lower respiratory infections. J Am Geriatr Soc 2004;52:51-8.

45. Livingston G, Lewis-Holmes E, Pitfield C, et al. Improving the end-of-life for people with dementia living in a care home: an intervention study. Int Psychogeriatr 2013;25:1849-58.

46. Mott PD, Barker WH. Hospital and medical care use by nursing home patients: the effect of patient care plans. J Am Geriatr Soc 1988;36:47-53. 